\title{
3.7 Exercise Systolic Blood Pressure Response and Incident Depressive Symptoms - The Maastricht Study
}

\author{
Tan Lai Zhou ${ }^{1,2,}$, Abraham Kroon ${ }^{1,2}$, Ronald Henry ${ }^{1,2}$, Annemarie Koster ${ }^{3,4}$, Pieter Dagnelie ${ }^{1,2}$, Hans Bosma ${ }^{3,4}$, \\ Marleen van Greevenbroek ${ }^{4,2}$, Carla van der Kallen ${ }^{4,2}$, Casper Schalkwijk ${ }^{4,2}$, Anke Wesselius ${ }^{4}$, Koen Reesink ${ }^{2,4}$, \\ Sebastian Köhler ${ }^{5,6}$, Miranda Schram ${ }^{1,2}$, Coen Stehouwer ${ }^{1,2}$, Thomas van Sloten ${ }^{1,2,7,8}$
}

\author{
${ }^{1}$ Maastricht University Medical Centre, Maastricht, The Netherlands \\ ${ }^{2}$ CARIM School for Cardiovascular Diseases, Maastricht, The Netherlands \\ ${ }^{3}$ CAPHRI Care and Public Health Research Institute, Maastricht, The Netherlands \\ ${ }^{4}$ Maastricht University, Maastricht, The Netherlands \\ ${ }^{5}$ Alzheimer Centre Limburg, Maastricht University Medical Centre, Maastricht, The Netherlands \\ ${ }^{6} \mathrm{MHeNs}$ School for Mental Health and Neuroscience, Maastricht, The Netherlands \\ ${ }^{7}$ Université Paris Descartes, Paris, France \\ ${ }^{8}$ INSERM Paris Cardiovascular Research Centre, Paris, France
}

\begin{abstract}
Background: Exaggerated exercise systolic blood pressure (SBP) is a modifiable risk factor associated with vascular dysfunction. Vascular dysfunction may contribute to the development of late-life depression, but the association between exercise SBP and incident depressive symptoms is unknown. Therefore, we investigated whether an exaggerated exercise SBP is associated with a higher risk of depressive symptoms over time.

Methods: Longitudinal data from the population-based Maastricht Study, with only individuals free of depressive symptoms at baseline included ( $n=2,121 ; 51.3 \%$ men; age $59.5 \pm 8.5$ years). Exercise SBP was measured at baseline with a submaximal exercise cycle test. We calculated a composite score of exercise SBP based on 4 standardized exercise SBP measures: SBP at moderate workload, SBP at peak exercise, SBP change per minute during exercise and SBP 4 minutes after exercise. Clinically relevant depressive symptoms were determined annually at follow-up and defined as a Patient Health Questionnaire score of $>=10$.
\end{abstract}

Results: After a mean follow-up of 3.9 years, 175 participants (8.3\%) had incident clinically relevant depressive symptoms. A1 standard deviation higher exercise SBP composite score was associated with a higher incidence of clinically relevant depressive symptoms (hazard ratio: 1.27 [95\% confidence interval: 1.04-1.54]). Results were adjusted for age, sex, education level, glucose metabolism status, lifestyle, cardiovascular risk factors, resting SBP and cardiorespiratory fitness.

Conclusion: A higher exercise SBP response is associated with a higher incidence of clinically relevant depressive symptoms.

(c) 2019 Association for Research into Arterial Structure and Physiology. Publishing services by Atlantis Press International B.V. This is an open access article distributed under the CC BY-NC 4.0 license (http://creativecommons.org/licenses/by-nc/4.0/). 